\title{
The Voronoi-Delaunay Approach for Modeling the Packing of Balls in a Cylindrical Container
}

\author{
V.A. Luchnikov ${ }^{1}$, N.N. Medvedev ${ }^{1}$, M.L. Gavrilova ${ }^{2}$ \\ ${ }^{1}$ Institute of Chemical Kinetics and Combustion, 630090 Novosibirsk, Russia \\ luchnik, nikmed@ns . kinetics.nsc.ru \\ ${ }^{2}$ Dept of Comp. Science, University of Calgary, AB, Canada, T2N1N4 \\ marina@cpsc.ucalgary.ca
}

\begin{abstract}
The paper presents an approach for calculation of the Voronoi network of a system of balls con ned inside a cylindrical container. We propose to consider a boundary of a container as one of the elements of the system. Then the Voronoi network can be built for a system containing non-spherical particles. An explicit formula to compute the coordinates of the Voronoi vertex between a three balls and a cylinder are obtained. The approach is implemented in $3 \mathrm{D}$ and tested on the models of balls packing with di erent structure.
\end{abstract}

\section{Introduction}

The Voronoi ideas, that are well known in mathematics and computer science, have been used extensively to solve many applied problems in physics, mechanics and chemistry [7,6]. Originally, the Voronoi-Delaunay approach in physics was applied to study the structure of disordered packing of balls and models of liquid and glasses [3]. The method is also a helpful tool for analysis of voids: empty spaces between atoms, where the Voronoi network plays the role of a navigation map [5]. This property of the Voronoi network has been used in studying various problems, in particular to model the permeability and the fluid flow through the packing of balls $[1,10]$.

Traditionally, the Voronoi-Delaunay method is applied to models with the periodic boundary conditions (used to simulate an infinite media) or to models with an open boundary (such as biological molecules. However, in many physicalchemical problems the boundary plays the determinative role. For example, a typical chemical reactor is a cylinder filled with the spherical granules. To simulate flows through the packing in the cylinder one should create an algorithm that takes into account the boundary of the system. From the mathematical point of view, the problem is similar to building the medial axes inside a cylindrical cavity containing balls. However, the known approaches to compute the medial axes inside a cavity $[8,9]$ are complicated and thus deem to be inefficient for the analysis of models with a large number of balls.

In this paper, we present an efficient approach for the calculation of the Voronoi network of a packing of balls inside a cylinder. We propose to consider a cylindrical boundary as an additional non-spherical element of the system, and provide an explicit formula to compute the coordinates of the Voronoi vertex between the three balls and a cylindrical boundary. The algorithm was implemented and tested in 3D for packings with different structure. 


\section{The algorithm}

One of the possible ways to compute the Voronoi network for a set of balls in a cylinder is to use the algorithm presented in [4], where the Voronoi network was calculated for 3D systems of straight lines and sphere-cylinders. The algorithm is based on the idea of the Delaunay empty sphere [7]. Let us assume that the empty sphere moves inside the system so that it touches at least three objects at any moment of time. In this case the center of the sphere moves along an edge of the 3D Voronoi network. If the distance from any point in the space to any object is expressed by explicit functions $d$, then the trajectory of the center of the Delaunay empty sphere can be computed numerically by performing the series of small shifts along the edge. The direction of the shift $\mathbf{v}$ is found from the equation: $\left.\left(\nabla d_{i} \cdot \mathbf{v}\right)\right|_{\mathbf{r}}=\left.\left(\nabla d_{j} \cdot \mathbf{v}\right)\right|_{\mathbf{r}}=\left.\left(\nabla d_{k} \cdot \mathbf{v}\right)\right|_{\mathbf{r}}$, where the indices $i, j, k$ enumerate the objects touched by the sphere. For a cylindrical wall we use the following distance function: $d_{c}=R_{c}-\left(x^{2}+y^{2}\right)^{1 / 2}$, where $R_{c}$ is the radius of the cylinder, $x$ and $y$ are the coordinates of a point inside the cylinder, provided the origin is on the axis of the cylinder. $d_{c}$ is a differentiable function of the coordinates, except for the axis of the cylinder. The advantage of this method is in its simplicity and versatility: it can be used to build the Voronoi network for the system of any convex non-spherical objects for which there is no explicit formula to compute the coordinates of the Voronoi vertex. However this approach is rather time consuming: as it was shown on a packing of balls of different radii this algorithm runs 20 times slower then the algorithm based on explicit calculation of the coordinates of the Voronoi vertex [4]. For the problem of balls in cylinder, we can find a formula for the Voronoi vertex explicitly.

\section{The Empty Sphere Problem}

The task is to inscribe a sphere between the cylinder and three balls, that are located inside the cylinder and do not intersect. For simplicity, assume the cylinder is vertical. Denote the sphere with the smallest radius as $\left(x_{4}, y_{4}, z_{4}, r_{4}\right)$. Choose the origin at the center of this sphere. Now, we apply the technique similar to one presented in [2]. We shrink all the balls by $r_{4}$, increase cylinder radius by $r_{4}$, and obtain the set of equations representing the condition that the inscribed sphere with the center $(x, y, z)$ and the radius $r$ touches three spheres and the cylinder:

$$
\left\{\begin{array}{l}
\left(x-x_{1}\right)^{2}+\left(y-y_{1}\right)^{2}=\left(r_{1}-r\right)^{2} \\
\left(x-x_{2}\right)^{2}+\left(y-y_{2}\right)^{2}+\left(z-z_{2}\right)^{2}=\left(r+r_{2}\right)^{2} \\
\left(x-x_{3}\right)^{2}+\left(y-y_{3}\right)^{2}+\left(z-z_{3}\right)^{2}=\left(r+r_{3}\right)^{2} \\
x^{2}+y^{2}+z^{2}=r^{2}
\end{array}\right.
$$

Here $\left(x_{1}, y_{1}, r_{1}\right)$ are the coordinates of the axis of the cylinder and its radius, and $\left(x_{i}, y_{i}, z_{i}, r_{i}\right), i=2,3$ are the coordinates and radii of the two remaining 
spheres. Subtracting the last equation from the first three we arrive to:

$$
\mathbf{A}\left[\begin{array}{l}
x \\
y \\
r
\end{array}\right]=\mathbf{b}, \mathbf{A}=\left[\begin{array}{lll}
x_{1} & y_{1} & -r_{1} \\
x_{2} & y_{2} & r_{2} \\
x_{3} & y_{3} & r_{3}
\end{array}\right], \mathbf{b}=\left[\begin{array}{c}
l_{1}-z^{2} \\
l_{2}-2 z z_{2} \\
l_{3}-2 z z_{3}
\end{array}\right],
$$

where $l_{1}=x_{1}{ }^{2}+y_{1}{ }^{2}-r_{1}^{2}, l_{i}=x_{i}{ }^{2}+y_{i}{ }^{2}+z_{i}{ }^{2}-r_{i}{ }^{2}, i=2,3$.

Solving the above system, we obtain:

$$
\begin{gathered}
x=\frac{1}{2|\mathbf{A}|}\left|\begin{array}{lll}
l_{1}-z^{2} & y_{1} & -r_{1} \\
l_{2}-2 z z_{2} & y_{2} & r_{2} \\
l_{3}-2 z z_{3} & y_{3} & r_{3}
\end{array}\right|, y=\frac{1}{2|\mathbf{A}|}\left|\begin{array}{lll}
x_{1} & l_{1}-z^{2} & -r_{1} \\
x_{2} & l_{2}-2 z z_{2} & r_{2} \\
x_{3} & l_{3}-2 z z_{3} & r_{3}
\end{array}\right|, \\
r=\frac{1}{2|\mathbf{A}|}\left|\begin{array}{lll}
x_{1} & y_{1} & l_{1}-z^{2} \\
x_{2} & y_{2} & l_{2}-2 z z_{2} \\
x_{3} & y_{3} & l_{3}-2 z z_{3}
\end{array}\right|,
\end{gathered}
$$

where $|\mathbf{A}|$ denotes the determinant of the matrix. When $|\mathbf{A}|=0$, we arrive to the degenerate case, i.e. there are infinitely many inscribed spheres. Thus, let us assume that $|\mathbf{A}| \neq 0$. The obtained expressions are substituted into the last equation $x^{2}+y^{2}+z^{2}-r^{2}=0$, which yields the 4 th degree polynomial of $z$ :

$$
a z^{4}+b z^{3}+c z^{2}+d z+e=0
$$

The coefficients in the above equation are in the form: $a=A_{x}{ }^{2}+A_{y}{ }^{2}-A_{r}{ }^{2}, b=$ $2\left(A_{x} B_{x}+A_{y} B_{y}-A_{r} B_{r}\right), c=B_{x}{ }^{2}+2 A_{x} C_{x}+B_{y}{ }^{2}+2 A_{y} C_{y}+4|\mathbf{A}|^{2}-B_{r}{ }^{2}-2 A_{r} C_{r}$, $d=2\left(B_{x} C_{x}+B_{y} C_{y}-B_{r} C_{r}\right), e=C_{x}{ }^{2}+C_{y}{ }^{2}-C_{r}{ }^{2}$, where

$$
\begin{gathered}
A_{x}=\left|\begin{array}{lll}
-1 & y_{1} & -r_{1} \\
0 & y_{2} & r_{2} \\
0 & y_{3} & r_{3}
\end{array}\right|, B_{x}=-2\left|\begin{array}{lll}
0 & y_{1} & -r_{1} \\
z_{2} & y_{2} & r_{2} \\
z_{3} & y_{3} & r_{3}
\end{array}\right|, C_{x}=\left|\begin{array}{lll}
l_{1} & y_{1} & -r_{1} \\
l_{2} & y_{2} & r_{2} \\
l_{3} & y_{3} & r_{3}
\end{array}\right|, \\
A_{y}=\left|\begin{array}{lll}
x_{1} & -1 & -r_{1} \\
x_{2} & 0 & r_{2} \\
x_{3} & 0 & r_{3}
\end{array}\right|, B_{y}=-2\left|\begin{array}{lll}
x_{1} & 0 & -r_{1} \\
x_{2} & z_{2} & r_{2} \\
x_{3} & z_{3} & r_{3}
\end{array}\right|, C_{y}=\left|\begin{array}{lll}
x_{1} & l_{1} & -r_{1} \\
x_{2} & l_{2} & r_{2} \\
x_{3} & l_{3} & r_{3}
\end{array}\right|, \\
A_{r}=\left|\begin{array}{lll}
x_{1} & y_{1} & -1 \\
x_{2} & y_{2} & 0 \\
x_{3} & y_{3} & 0
\end{array}\right|, B_{r}=-2\left|\begin{array}{lll}
x_{1} & y_{1} & 0 \\
x_{2} & y_{2} & z_{2} \\
x_{3} & y_{3} & z_{3}
\end{array}\right|, C_{r}=\left|\begin{array}{lll}
x_{1} & l_{1} & l_{1} \\
x_{2} & l_{2} & l_{2} \\
x_{3} & l_{3} & l_{3}
\end{array}\right| .
\end{gathered}
$$

The equation is then solved for $z$. The final answer is:

$$
\begin{gathered}
x_{f}=\frac{\left(A_{x} z^{2}+B_{x} z+C_{x}\right)}{2|\mathbf{A}|}+x_{4}, y_{f}=\frac{\left(A_{y} z^{2}+B_{y} z+C_{y}\right)}{2|\mathbf{A}|}+y_{4}, \\
r_{f}=\frac{\left(A_{r} z^{2}+B_{r} z+C_{r}\right)}{2|\mathbf{A}|}-r_{4}, z_{f}=z+z_{4} .
\end{gathered}
$$

Up to four solutions are possible. However, the solutions with imaginary and negative $r$ are non-physical and omitted. 


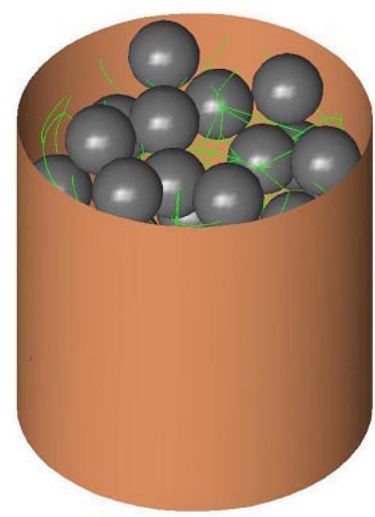

(a)

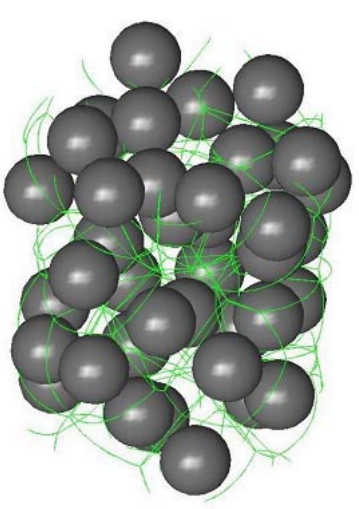

(b)

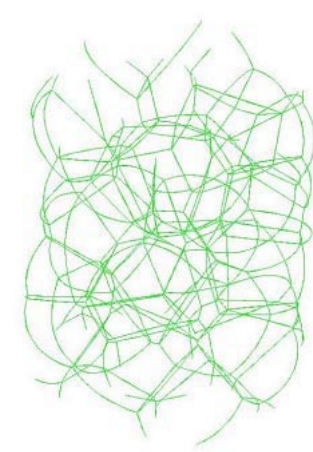

(c)

Figure 1: A cylinder with the balls (a), the arrangement of the balls in the cylinder (b), the Voronoi network (edges of the Voronoi diagram) taking into consideration the cylinder (c).

The algorithm for Voronoi network calculation was implemented in Fortran. Fig. 1 illustrate its application for a system of 40 balls with equal radii $r=0.2$ in a cylinder of radii $r_{c}=2.0$. One can note that the Voronoi edges that are at the center of the cylinder are segments of straight lines. They are edges of the standard Euclidean Voronoi diagram. While the edges at the cylinder surface are curved. This situation is typical for systems with the non-spherical particles (the edges are on the intersection of curved quadratic surfaces). The algorithm was also tested on a system representing dense packing of 300 Lennard-Jones atoms with different structure. Two models: one is a model with disordered packing obtained by Monte-Carlo relaxation in a cylinder with a fixed value of the diameter $D=6 \sigma$ (where $\sigma$ is the parameter of the Lennard-Jones potential) and the other model with the crystalline-like structure (made by slightly varying the diameter of cylinder). The reslts show that the largest channels (Voronoi network bonds with the largest bottle-necks) occur near to the wall of the cylinder. This is an anticipated result, since a flow of liquid through a packing of balls is inhomogeneous and the main streams are at the cylinder wall. It was also noted that a fraction of large channels along the wall is higher for the model with the disordered packing than for the crystalline-like model.

\section{Future Work and Acknowledgments}

One of the possible directions of the future research is extension of the method to handle different types of curvlinear boundaries and the experimentation with 
physical systems built inside given boundaries. The work was supported in part by SB RAS No.46, RFFI No.01-03-32903 and UCRS grants. We also would like to thank Dr. Annie Gervois for helpful comments and suggestions.

\section{References}

[1] Bryant, S. and Blunt, M. , Phys. Rev. A, 46(4) (1992) 2004

[2] Gavrilova, M. and Rokne, J. Swap conditions for dynamic Voronoi diagram for circles and line segments, Comp-Aided Geom. Design, 16 (1999) 89-106

[3] Finney, J. Random packings and the structure of simple liquids. Roy.Soc.London, 319 (1970) 479-495

[4] Luchnikov, V.A., Medvedev,N.N., Oger, L. and Troadec, J.-P. The VoronoiDelaunay analysis of voids in system of nonspherical particles. Phys.Rev.E. 59(6), (1999) 7205-7212

[5] Medvedev, N.N. Computational porosimetry, in Voronoi's impact on modern science. Ed. P. Engel, H. Syta, Inst. of Math., Kiev., (1998) 164-175

[6] Medvedev, N.N. Voronoi-Delaunay method for non-crystalline structures, SB Russian Academy of Science, Novosibirsk, (2000)

[7] Okabe, A., Boots, B., Sugihara, K. Spatial tesselation concepts and applications of Voronoi diagrams, J. Wiley \& Sons, Chichester, England (1992)

[8] Rowe, N.C. Obtaining Optimal Mobile-Robot Paths with Non-Smooth Anisotropic Cost Functions, J. Robot. Res, 16(3) (1997) 375-399

[9] Sherbrooke E. C., Patrikalakis N.M. and Brisson, N. An Algorithm for the Medial Axis Transform of 3D Polyhedral Solids, IEEE Trans. Visualiz. Comp. Graph, 2(1) (1996) 45-61

[10] Thompson, K.E and Fogler, H.S. Modelling flow in disordered packed bed from pore-scale fluid mechanics. AICHE Journal, 43(6) (1997) 1377-1389 